\title{
Orthomodular posets are algebras over bounded posets with involution
}

\author{
Gejza Jenča
}

the date of receipt and acceptance should be inserted later

\begin{abstract}
We prove that there is a monadic adjunction between the category of bounded posets with involution and the category of orthomodular posets.
\end{abstract}

Mathematics Subject Classification (2010) Primary 03G12, Secondary: 06F20, 81P10

Keywords orthomodular poset, monadic functor

\section{Introduction}

Effect algebras were introduced in Foulis and Bennett (1994) as (at that point in time) the most general version of quantum logics. The motivating example was the set of all Hilbert space effects, a notion that plays an important role in quantum mechanics Ludwig (1983); Busch et al. (1996). An equivalent definition was given independently in Kôpka and Chovaned (1994). Later it turned out that both groups of authors rediscovered the definition given already in Giuntini and Greuling (1989).

In Kalmbach (1977) the following theorem was proved.

Theorem 1.1 Every bounded lattice $L$ can be embedded into an orthomodular lattice $K(L)$.

The proof of the theorem is constructive, $K(L)$ is known under the name Kalmbach extension or Kalmbach embedding. In Mavet and Navara (1995), authors proved that Theorem 1.1 can be generalized: every bounded poset $P$ can be embedded in an orthomodular poset

G. Jenča

Department of Mathematics and Descriptive Geometry

Faculty of Civil Engineering, Slovak University of Technology,

Slovak Republic

gejza.jenca@stuba.sk
$K(P)$. In fact, by Harding (2004), this $K$ is then left adjoint to the forgetful functor from orthomodular posets to bounded posets. This adjunction gives rise to a monad on the category of bounded posets, which we call the Kalmbach monad.

For every monad $(T, \eta, \mu)$ on a category $\mathcal{C}$, there is an Eilenberg-Moore category $\mathcal{C}^{T}$ (sometimes called the category of algebras over $T$ or the category of modules over $T$ ). The category $\mathcal{C}^{T}$ comes equipped with a canonical adjunction between $\mathcal{C}$ and $\mathcal{C}^{T}$ and this adjunction gives rise to the original monad $T$ on $\mathcal{C}$. A functor equivalent to a right adjoint $\mathcal{C}^{T} \rightarrow \mathcal{C}$ is called monadic.

It was proved in Jenča (2015) that the EilenbergMoore category for the Kalmbach monad is isomorphic to the category of effect algebras. In other words, the forgetful functor from the category of effect algebras to the category of bounded posets is monadic. Later, it was proved in Jenča (2020) that the forgetful functor from the category of pseudo-effect algebras (see Dvurečenskij and Vetterlein (2001)) to the category of bounded posets is monadic. Recently, it was proved in van de Wetering (2021) that both $\omega$-complete effect algebras and $\omega$-complete effect monoids can be represented as categories of algebras over the category of bounded posets.

On the other hand, it clearly follows from Jenča (2015) that the right adjoint functor from the category of orthomodular posets to the category of bounded posets is not monadic. Indeed, this right adjoint gives rise to the Kalmbach monad and the Eilenberg-Moore category for the Kalmbach monad is the category of effect algebras which is clearly not equivalent to the category of orthomodular posets.

This leads to a natural question: is the category of orthomodular posets equivalent to a category of algebras for some nontrivial monad? In the present paper, 
we answer this question in the positive. We prove that the forgetful functor from the category of orthomodular posets to the category of bounded posets equipped with involution is monadic.

\section{Preliminaries}

We assume familiarity with basics of category theory, see MacLane (1971); Awodey (2006); Riehl (2016) for reference.

\subsection{Posets and bounded posets}

Pos is the usual category of posets equipped with isotone maps. A bounded poset is a structure $(P, \leq, 0,1)$ such that $\leq$ is a partial order on $P$ and $0,1 \in P$ are the bottom and top elements of $(P, \leq)$, respectively.

Let $P_{1}, P_{2}$ be bounded posets. A map $f: P_{1} \rightarrow P_{2}$ is a morphism of bounded posets if and only if it satisfies the following conditions.

- $f$ is isotone.

$-f(1)=1$ and $f(0)=0$.

The category of bounded posets is denoted by BPos.

\subsection{Bounded posets with involution}

An involution on a poset $P$ is mapping ' $: P \rightarrow P$ satisfying the following conditions.

- For all $x, y \in P, x \leq y$ if and only if $y^{\prime} \leq x^{\prime}$.

- For all $x \in P, x^{\prime \prime}=x$.

Note that every bounded poset with involution $(P, \leq$ $\left.,^{\prime}, 0,1\right)$ satisfies $0^{\prime}=1$. Indeed, for every $x \in P, 0 \leq x^{\prime}$ implies $x^{\prime \prime}=x \leq 0^{\prime}$, so $0^{\prime}$ is the greatest element of $P$.

Two elements $x, y$ of a bounded poset with involution are said to be orthogonal (in symbols $x \perp y$ ), if $x \leq y^{\prime}$. Note that $\perp$ is a symmetric relation and that for all $x \in P, x \perp 0$.

The objects of the category BPosInv are bounded posets with involution (sometimes called involutive bounded posets). A mapping $f: P \rightarrow Q$ between two bounded posets with involution is a morphism in BPosInv if and only if the following conditions are satisfied.

- $f$ is a morphism of bounded posets.

- For all $x \in P, f\left(x^{\prime}\right)=(f(x))^{\prime}$.

\subsection{Effect algebras}

An effect algebra is a partial algebra $(E,+, 0,1)$ with a binary partial operation + and two nullary operations 0,1 satisfying the following conditions.

(E1) If $a+b$ is defined, then $b+a$ is defined and $a+b=$ $b+a$.

(E2) If $a+b$ and $(a+b)+c$ are defined, then $b+c$ and $a+(b+c)$ are defined and $(a+b)+c=a+(b+c)$.

(E3) For every $a \in E$ there is a unique $a^{\prime} \in E$ such that $a+a^{\prime}$ exists and $a+a^{\prime}=1$.

(E4) If $a+1$ is defined, then $a=0$.

In an effect algebra $E$, we write $a \leq b$ if and only if there is $c \in E$ such that $a+c=b$. It is easy to check that for every effect algebra $\left(E, \leq,{ }^{\prime}, 0,1\right)$ is a bounded poset with involution. Moreover, it is possible to introduce a new partial operation $-; b-a$ is defined if and only if $a \leq b$ and then $a+(b-a)=b$. It can be proved that, in an effect algebra, $a+b$ is defined if and only if $a \perp b$.

Let $E_{1}, E_{2}$ be effect algebras. A map $f: E_{1} \rightarrow E_{2}$ is called a morphism of effect algebras if and only if it satisfies the following conditions.

$-f(1)=1$.

- If $a+b$ exists, then $f(a)+f(b)$ exists and $f(a+b)=$ $f(a)+f(b)$.

The category of effect algebras is denoted by EA.

We note that every morphism of effect algebras is isotone and preserves 0,1 and the involution, hence every morphism of effect algebras is a morphism of the underlying bounded posets with involution. Moreover, every morphism of effect algebras preserves the partial operation - .

\subsection{Orthomodular posets}

An orthomodular poset is a bounded poset with involution $\left(A, \leq,^{\prime}, 0,1\right)$ satisfying the following conditions, for all $x, y \in A$.

(OMP1) $x \wedge x^{\prime}=0$.

(OMP2) If $x \perp y$, then $x \vee y$ exists.

(OMP3) If $x \leq y$, then $x \vee\left(x \vee y^{\prime}\right)^{\prime}=y$.

Let $A_{1}, A_{2}$ be orthomodular posets. A map $f: A_{1} \rightarrow$ $A_{2}$ is called a morphism of orthomodular posets if and only if it is a morphism of bounded posets with involution such that for all $x \perp y$ in $A_{1}, f(x) \perp f(y)$ and $f(x \vee y)=f(x) \vee f(y)$.

Let $A$ be an orthomodular poset. A subset $B \subseteq A$ is a subalgebra of $A$ if and only if $0,1 \in B, B$ is closed with respect to ' and for all $x, y \in B$ such that $x \perp y$, $x \vee y \in B$. 
The category of orthomodular posets is denoted by OMP. If $A$ is an orthomodular poset, then we may introduce a partial + operation on $A$ by the following rule: $x+y$ exists if and only if $x \perp y$ and then $x+y:=$ $x \vee y$. The resulting structure is then an effect algebra. This gives us the object part of an evident full and faithful functor $\mathbf{O M P} \rightarrow \mathbf{E A}$.

Proposition 2.1 The category $\mathbf{O M P}$ is small-complete.

Proof It is easy to check that a product of every family of orthomodular posets can be constructed as a product of underlying bounded posets, the involution ' is defined pointwise. For a parallel pair of morphisms $f, g: A \rightarrow B$ in OMP, their equalizer is the inclusion of a subalgebra $E=\{x \in A: f(x)=g(x)\}$ into $A$. Since the category OMP has all products and all equalizers, it has all small limits.

The following functors will be used in what follows.

\subsection{The functor $U:$ BPosInv $\rightarrow$ Pos}

$U$ is just the forgetful functor that takes every object of BPosInv to its underlying poset.

\subsection{The functor $\perp:$ BPosInv $\rightarrow$ Pos}

The orthogonality relation on the objects of BPosInv can be exhibited as a functor from BPos to Pos, as follows. For every bounded poset with involution $P$, $\perp(P)$ is the order ideal in the poset $U(P) \times U(P)$ given by the rule

$$
\perp(P)=\left\{(x, y) \in U(P) \times U(P) \mid x \leq y^{\prime}\right\} .
$$

For a morphism $f: P \rightarrow Q$ in BPosInv, $\perp(f): \perp(P) \rightarrow$ $\perp(Q)$ is given by $\perp(f)(x, y)=(f(x), f(y))$.

\subsection{The functor $\Perp:$ BPosInv $\rightarrow$ Pos}

For every bounded poset with involution $P, \Perp(P)$ is the order ideal in the poset $U(P) \times U(P) \times U(P)$ given by the rule

$$
\begin{aligned}
\Perp(P)=\{(x, y, z) \in U(P) \times U(P) \times U(P) \mid \\
x \perp y \text { and } x \perp z \text { and } y \perp z\} .
\end{aligned}
$$

On morphisms, $\Perp$ is defined coordinatewise, similarly as we did for $\perp$.

\subsection{The functor $I:$ Pos $\rightarrow$ Pos}

For every poset $P$, let us write $I(P)$ for the set of comparable pairs $\{(a, b) \in P \times P: a \leq b\}$ and partially order $I(P)$ by the rule $(a, b) \leq(c, d)$ if and only if $c \leq a \leq b \leq d$. Note that the elements of $I(P)$ can be identified with closed intervals of $P$, ordered by inclusion. We shall write $[a \leq b]$ or $[b \geq a]$ for the element $(a, b)$ of $I(P)$. The construction $P \mapsto I(P)$ can be made into a functor Pos $\rightarrow$ Pos by the rule $I(f)([a \leq b])=[f(a) \leq f(b)]$.

\subsection{General adjoint functor theorem}

Adjoint functor theorems give conditions under which a continuous functor $G$ has a left adjoint $F$. This allows us to avoid construction of the functor $F$, which is sometimes a difficult endeavor.

Theorem 2.1 (MacLane, 1971, Theorem V.6.2) Freyd (1964) Given a locally small, small-complete category $\mathcal{D}$, a functor $G: \mathcal{D} \rightarrow \mathcal{C}$ is a right adjoint if and only if $G$ preserves small limits and satisfies the following

Solution Set Condition: for each object $X$ of $\mathcal{C}$ there is a set $I$ and an I-indexed family of arrows $h_{i}: X \rightarrow$ $G\left(A_{i}\right)$ such that every arrow $h: X \rightarrow G(A)$ can be written as a composite $h=G(j) \circ h_{i}$ for some $j: A_{j} \rightarrow A$.

\subsection{Beck's monadicity theorem}

A functor $G: \mathcal{D} \rightarrow \mathcal{C}$ is monadic if and only if it is equivalent to the forgetful functor from the category of algebras $\mathcal{C}^{T}$ to $\mathcal{C}$ for a monad $T$ on $\mathcal{C}$. A colimit (or a limit) in a category $\mathcal{C}$ is absolute if and only if it is preserved by every functor with domain $\mathcal{C}$.

Theorem 2.2 (MacLane, 1971, Theorem VI.7.1)Beck (1967) A functor

$$
G: \mathcal{D} \rightarrow \mathcal{C}
$$

is monadic if and only if $G$ is a right adjoint and $G$ creates coequalizers for those parallel pairs $f, g: A \rightarrow B$ in $\mathcal{D}$, for which

$$
G(A) \underset{G(g)}{\stackrel{G(f)}{\longrightarrow}} G(B)
$$

has an absolute coequalizer in $\mathcal{C}$.

Beck's monadicity theorem is a device that allows us to prove that a functor is monadic without having to explicitly describe the monad $T$ on $\mathcal{C}$ arising from the adjunction, describe its category of algebras $\mathcal{C}^{T}$ and to prove that $\mathcal{C}^{T}$ is equivalent to $\mathcal{D}$. 


\section{The result}

The aim of this paper is to prove that the obviously defined forgetful functor from OMP to BPosInv is a monadic right adjoint. To do this, we need to characterize orthomodular posets as bounded posets with involution equipped with an additional structure.

Proposition 3.1 Let $A$ be a bounded poset with involution equipped with two partial operations + and such that

$-x+y$ is defined if and only if $x \perp y$ and

$-y-x$ is defined if and only if $x \leq y$.

Suppose that the mappings

$$
+: \perp(A) \rightarrow U(A)
$$

and

$$
-: I U(A) \rightarrow U(A)
$$

corresponding to these partial operations are isotone and that the following conditions are satisfied.

(A0) For all $a \in A, a \perp 0$ and $a+0=a$.

(A1) For all $a, b \in A, a \perp b$ implies that $b \perp a$ and then $a+b=b+a$

(A2) For all $a, b, c \in A$, if $a \perp b$ and $b \perp c$ and $a \perp c$, then $a \perp(b+c)$ and $(a+b) \perp c$ and $a+(b+c)=$ $(a+b)+c$.

(A3) For all $a, b \in A$ such that $a \perp b, a+b \geq a$ and $(a+b)-a=b$.

(A4) For all $a \in A, a+a^{\prime}=1$.

Then $A$ is an orthomodular poset with $x \vee y=x+y$, for all $x \perp y$.

Moreover, for every orthomodular poset $A$, putting $x+y=x \vee y$ and $x-y=x \wedge y^{\prime}$ gives us a pair of partial operations satisfying these conditions.

Proof The fact that every orthomodular poset satisfies these conditions is well known. By (Foulis and Bennett, 1994, Theorem 5.3), an effect algebra is an orthomodular poset with $a \vee b=a+b$ if and only if it satisfies (A2). So it remains to prove that $A$ is an effect algebra. The conditions (E1) and (A1) are the same. To prove (E2), suppose that $a, b, c \in A$ are such that $a \perp b$ and $(a+b) \perp c$, that means $a+b \leq c^{\prime}$. From (A0) and the fact that + is isotone we obtain $a=(a+0) \leq$ $(a+b) \leq c^{\prime}$, hence $a \perp c$ and (similarly) $b \perp c$. By (A3), $a \perp(b+c)$ and $(a+b)+c=a+(b+c)$. The existence part of (E3) is exactly (A4). To prove the uniqueness part of (E3), suppose that $1=a+b_{1}=a+b_{2}$. By (A3), it then follows that

$$
b_{1}=\left(a+b_{1}\right)-a=1-a=\left(a+b_{2}\right)-a=b_{2} .
$$

To prove (E4), note that $a+1$ defined means that $a \perp 1$, which means $a \leq 1^{\prime}=0$ and hence $a=0$.
Since every orthomodular poset is a bounded poset with involution and we defined a morphism of orthomodular poset as a special type of morphism of the underlying bounded posets with involution, there is an obviously defined forgetful functor $G$ : OMP $\rightarrow$ BPosInv.

The main result of the present paper follows.

Theorem 3.1 The forgetful functor

$$
G: \text { OMP } \rightarrow \text { BPosInv }
$$

is monadic.

Proof Let us apply Theorem 2.1 to prove that $G$ is a right adjoint functor. By Proposition 2.1, OMP is small-complete. It is easy to check that $G$ preserves all small limits. Let us check the Solution Set Condition. Let $P$ be a bounded poset with involution. Let $\mathcal{W}_{P}$ be a set of bounded posets with involution such that for every bounded poset with involution $V$ with $\operatorname{card}(P) \leq$ $\operatorname{card}(V) \leq \max \left(\operatorname{card}(P), \aleph_{0}\right)$, there is a $W \in \mathcal{W}_{P}$ such that $W$ is isomorphic to $V$. Consider the family $\mathcal{H}_{P}=$ $\left\{h_{i}\right\}_{i \in I}$ of all BPosInv-morphisms $h_{i}: P \rightarrow G\left(A_{i}\right)$, where $A_{i}$ is an orthomodular poset and $G\left(A_{i}\right) \in \mathcal{W}_{P}$. For every BPosInv-morphism $h: P \rightarrow G(A)$, the cardinality of the subalgebra $B$ of $A$ that is generated by the range of $h$ is bounded below by $\operatorname{card}(P)$ and above by $\max \left(\operatorname{card}(P), \aleph_{0}\right)$. Write $j: B \rightarrow A$ for the embedding of the subalgebra $B$ into $A$. Clearly, $h=G(j) \circ h_{i}$ for some $h_{i} \in \mathcal{H}_{P}$. Since $G$ preserves small limits and the Solution Set Condition is satisfied, $G$ is a right adjoint.

We have proved that $G$ is a right adjoint, so we may apply Theorem 2.2. Let $A, B$ be orthomodular posets, let $f, g: A \rightarrow B$ be morphisms of orthomodular posets. Suppose that

$G(A) \underset{G(g)}{\stackrel{G(f)}{\longrightarrow}} G(B) \stackrel{q}{\longrightarrow} Q$

is an absolute coequalizer. Assuming this, we need to prove that there is a unique morphism of orthomodular posets $\widehat{q}: B \rightarrow \widehat{Q}$ such that

$$
A \underset{g}{\stackrel{f}{\longrightarrow}} B \stackrel{\widehat{q}}{\longrightarrow} \widehat{Q}
$$

is a coequalizer in OMP and $Q=G(\widehat{Q}), q=G(\widehat{q})$. Let us prove that such $\widehat{q}$ exists. We use the fact that (3.1) is an absolute coequalizer to equip the bounded poset with involution $Q$ with a structure of an orthomodular poset, in the sense of Proposition 3.1. Then we prove that $q$ comes from a morphism of orthomodular posets. Finally, we prove that this morphism of orthomodular posets is a coequalizer of $f, g$ in $\mathbf{O M P}$. 
Let $A$ be an orthomodular poset. Consider Proposition 3.1 the partial operation $+_{A}$ on $A$ is defined for pairs $a, b \in A$ with $a \perp b$, so we may represent it as an isotone mapping (in other words, a morphism in Pos) from the poset $\perp G(A)$ to the poset $U G(A)$. Similarly, the partial operation $-{ }_{A}$ on $A$ can be represented by an Pos-morphism $-{ }_{A}: I U G(A) \rightarrow U G(A)$. Moreover, every morphism $h: X \rightarrow Y$ of orthomodular posets preserves the partial operations + and - , that means that the diagrams

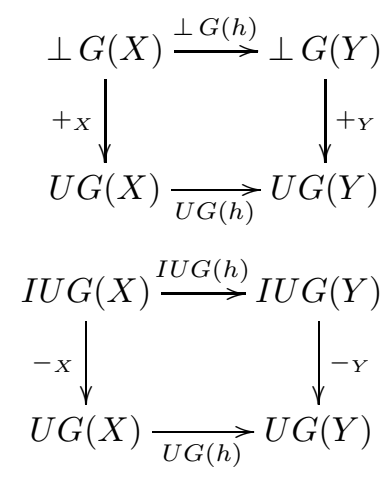

commute. Hence + and - are natural transformations in the category of functors [OMP, Pos].

Consider the diagram

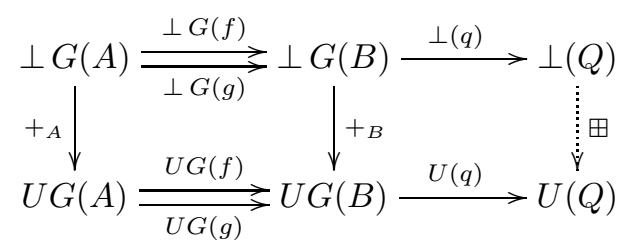

Since $f$ and $g$ are morphisms of orthomodular posets, the naturality of + implies that both $f$ and $g$ left-hand squares in (3.5) commute. From this and from the fact that the bottom row is a coequalizer, it follows that the morphism $+_{B} \circ U(q)$ coequalizes the top pair of parallel arrows. Indeed,

$$
\begin{aligned}
U(q) \circ+_{B} \circ \perp G(f) & =U(q) \circ U G(f) \circ+_{A} \\
& =U(q) \circ U G(g) \circ+_{A} \\
& =U(q) \circ+_{B} \circ \perp G(g) .
\end{aligned}
$$

Since (3.1) is an absolute coequalizer, the top row in (3.5) is a coequalizer. Since the top row in (3.5) is a coequalizer, there is a unique arrow $\boxplus: \perp(Q) \rightarrow U(Q)$ making the right-hand square of (3.5) commute. This way, we equipped the involutive bounded poset $Q$ with a partial binary operation $\boxplus$, defined for all orthogonal pairs of elements of $Q$.
In an analogous way, we may the use the diagram

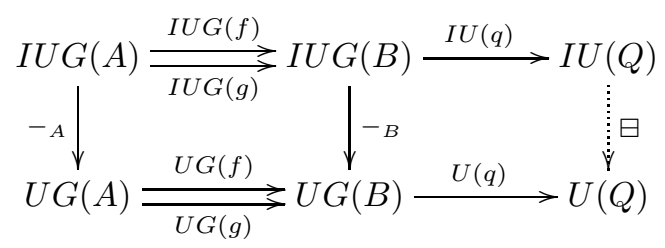

to define a partial operation $\boxminus: I U(Q) \rightarrow U(Q)$ on $Q$.

Let us prove that these partial operations on $Q$ satisfy the conditions of Proposition 3.1. We will proceed as follows: we will show that the fact that $X$ is an orthomodular poset can be expressed by encoding the conditions (A0)-(A4) by means of functors and natural transformations. For (A0), we will then give a detailed proof that $Q$ satisfies (A0). After that, we will observe that the proof for the remaining conditions can be given in a similar way.

(A0) For every bounded poset $P$ with involution, there is an isotone mapping

$$
\left(0,{ }_{-}\right)_{P}: U(P) \rightarrow \perp(P)
$$

that maps every $a \in U(P)$ to the pair $(0, a) \in$ $\perp(P)$. This $o b$ (BPosInv) indexed family of arrows forms a natural transformation from $U$ to $\perp$. The $(\mathrm{A} 0)$ property means that for every orthomodular poset $X$, the diagram

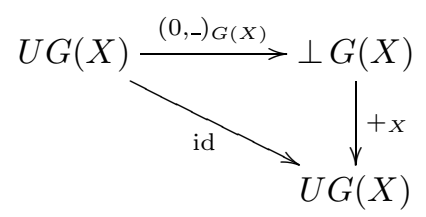

commutes. Therefore, $+\circ\left(\left(0,{ }_{-}\right) G\right)=\mathrm{id}_{U G}$ in the category of functors [OMP, Pos]. Equivalently, the diagram<smiles>[Te][Te]</smiles>

in the category [OMP, Pos] commutes.

(A1) For every bounded poset with involution $P$, there is a poset automorphism $s_{P}: \perp(P) \rightarrow \perp(P)$ given by the rule $s_{P}(a, b)=(b, a)$. Moreover, the family of morphisms $s_{\text {_ }}$ indexed by the objects of BPosInv forms a natural transformation

$$
s: \perp \rightarrow \perp
$$

in the category of functors [OMP, Pos]. 
The axiom (A1) means, that for every orthomodular poset $X$, the diagram

$$
\perp G(X) \stackrel{s_{G(X)}}{\longrightarrow} \perp G(X)
$$

commutes. This implies that $+\circ(s G)=+$ in the category of functors [OMP, Pos].

(A2) For every orthomodular poset $X$, there are isotone mappings

$$
r_{X}, l_{X}: \Perp G(X) \rightarrow \perp G(X)
$$

given by the rules

$$
r_{X}(a, b, c)=(a, b+c) \quad l_{X}(a, b, c)=(a+b, c)
$$

The families of Pos-morphisms $r_{-}$and $l_{-}$indexed by objects of OMP form a pair of natural transformations

$$
r, l: \Perp G \rightarrow \perp G
$$

in the category of functors [OMP, Pos]. The property (A3) then means that for every orthomodular poset $X$, the diagram

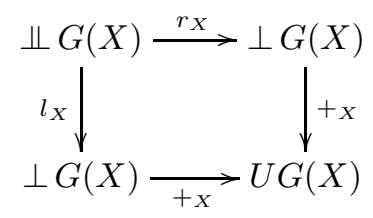

commutes, so $+\circ r=+\circ l$ in the category of functors [OMP, Pos].

(A3) For every orthomodular poset $X$, there is a mapping $p_{X}: \perp G(X) \rightarrow I U G(X)$ given by the rule

$$
p_{X}(a, b)=(a+b, a)
$$

Let $\pi_{X}: \perp G(X) \rightarrow U G(X)$ be the projection to the first entry, given by the rule $\pi(a, b)=a$. Again, the families $p_{-}$and $\pi_{-}$indexed by objects of OMP are natural transformations in the category of functors [OMP, Pos].

By (A3), the diagram

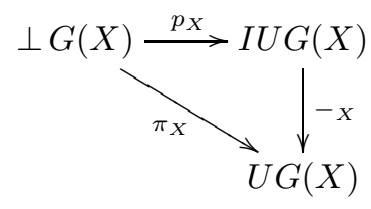

commutes, meaning that $-\circ p=\pi$ in the category [OMP, Pos].
(A4) To express this property using functors and natural transformations, we need to change the target category of our functors from Pos to Set. This appears to necessary because the mapping $a \mapsto\left(a, a^{\prime}\right)$ is not isotone.

Write $S:$ Pos $\rightarrow$ Set for the straightforwardly defined functor that takes every poset to its underlying set. For every orthomodular poset $X$, there is an morphism of sets $c_{X}: S U G(X) \rightarrow S \perp G(X)$ given by the rule $c_{X}(a)=\left(a, a^{\prime}\right)$. The property (A4) then means that the diagram

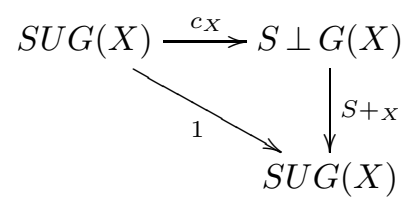

commutes; here, 1: $S U G(X) \rightarrow S G(X)$ denotes the constant mapping with value $1 \in S G(X)$. Therefore, $c \circ(S+)=1$ in the category of functors [OMP, Set].

Let us prove in detail that the partial operation $\boxplus$ on $Q$ satisfies (A0). Consider the diagram

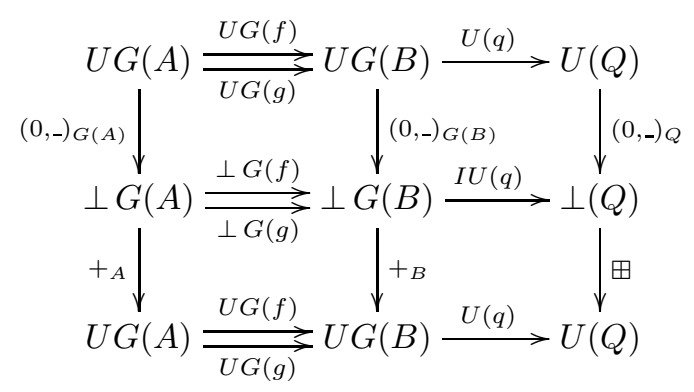

Since (3.7) commutes, both left and middle verticals of (3.11) compose to identity, therefore

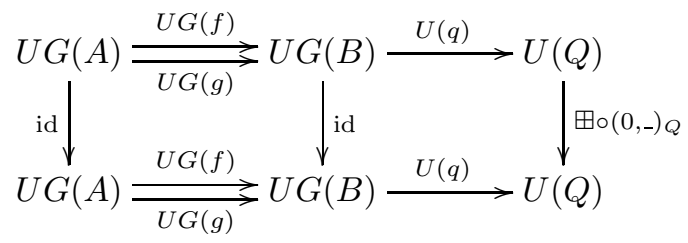

commutes. Note that if we replace the rightmost arrow in (3.12) by id, the diagram still commutes. However, by an analogous argument that we used to define + on $Q$, the rightmost vertical arrow in (3.12) is unique, meaning that the diagram

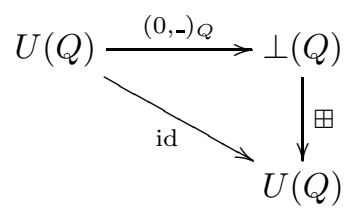


commutes. Therefore, $\boxplus \circ\left(0,{ }_{-}\right)_{Q}=i d_{U(Q)}$ or, in other words, for all $x \in Q, 0 \boxplus x$ is defined and $0 \boxplus x=x$. Thus, the partial operation $\boxplus$ on $Q$ satisfies the condition $(\mathrm{A} 0)$.

The reader should now observe that the essence of our proof that $\boxplus$ satisfies the contition (A0) lies in the fact that we can represent the condition (A0) by the commutative diagram (3.8) containing functors of the type $\_\circ G:$ OMP $\rightarrow$ Pos and natural transformations among them. A routine argument then allows us to use the fact that the orthomodular posets $A$ and $B$ satisfy (A0) to show that (3.13) commutes, hence the partial operation $\boxplus$ on $Q$ satisfies the condition (A0). In the previous part of the proof we have demonstrated that it is possible to express the remaining conditions (A1)-(A4) by a commutative diagram in [OMP, Pos] or [OMP, Set]. We may thus prove in an analogous way that the partial operations $\boxplus$ and $\boxminus$ on $Q$ satisfy the conditions (A1)-(A4) so we skip the this part of the proof.

We know that the partial operations $\boxplus$ and $\boxminus$ we defined on $Q$ satisfy the conditions (A0)-(A4). In other words, there is a orthomodular poset $\widehat{Q}$ such that $Q=$ $G(\widehat{Q})$. Moreover, the BPosInv-morphism $q: G(B) \rightarrow$ $Q=G(\widehat{Q})$ satisfies $q(x+y)=q(x) \boxplus q(y)$, for all $x \perp y$ in $B$, because the right-hand square of (3.5) commutes. That means, there is a morphism of orthomodular posets $\widehat{q}: B \rightarrow \widehat{Q}$ such that $q=G(\widehat{q})$. With this fact in mind, we may now observe that the diagram (3.5) means that $\widehat{q} \circ f=\widehat{q} \circ g$ in OMP and since the orthomodular poset structure on $Q$ arising from the diagrams (3.5) and (3.6) is unique, we see that $\widehat{Q}$ is unique. Uniqueness of the morphism $\widehat{q}$ follows from the fact that $G$ is a faithful functor.

Let us prove that $\widehat{q}$ is a coequalizer of the pair $f, g$ in OMP. Let $h: B \rightarrow C$ be a morphism of orthomodular posets such that $h \circ f=h \circ g$. Since the diagram (3.1) is a coequalizer in BPosInv, there is a unique morphism of bounded posets with involution $e: G(\widehat{Q}) \rightarrow G(C)$ such that $e \circ G(\widehat{q})=e \circ q=G(h)$. It remains to prove that this $e$ preserves the partial operation $\boxplus$ on $Q$. Consider the following diagram:

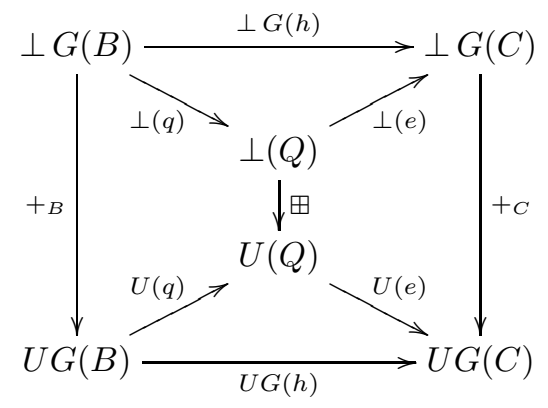

We need to prove that the right-hand square in (3.14) commutes. By the commutativity of the diagram (3.5), we already know that the left hand square in (3.14) commutes. moreover, as $G(h)=e \circ G(\widehat{q})$ we see that

$$
\perp G(h)=\perp(e \circ G(\widehat{q}))=\perp(e) \circ \perp G(\widehat{q})=\perp(e) \circ \perp(q)
$$

so the top triangle in (3.14) commutes. Similarly, we can prove

$$
U G(h)=U(e) \circ U(q)
$$

so the bottom triangle in (3.14) commutes. Since $h$ is a morphism of orthomodular posets, the outer square of (3.14) commutes. Therefore,

$$
\begin{aligned}
& +_{C} \circ \perp(e) \circ \perp(q)=+_{C} \circ \perp G(h)= \\
& U G(h) \circ+_{B}=U(e) \circ U(q) \circ+_{B}=U(e) \circ \boxplus \circ \perp(q)
\end{aligned}
$$

Since the top row in (3.5) is a coequalizer, $\perp(q)$ is a coequalizing arrow and thus an epimorphism. Therefore, (3.15) implies that $+_{C} \circ \perp(e)=U(e) \circ \boxplus$ and we see that the right-hand square of (3.14) commutes.

\section{Further research}

Sice $G$ is a right adjoint, there is a functor

$$
F: \text { BPosInv } \rightarrow \text { OMP }
$$

left adoint to $G$. It would be interesting to describe this functor, in an explicit way.

\section{Declarations}

Funding: This research is supported by grants VEGA $2 / 0142 / 20$ and $1 / 0006 / 19$, Slovakia and by the Slovak Research and Development Agency under the contracts APVV-18-0052 and APVV-20-0069.

Conflict of interest/Competing interest: None.

Availability of data and material: Not applicable. Code availability: Not applicable.

Authors' contributions: There is a single author.

\section{References}

Awodey S (2006) Category Theory. No. 49 in Oxford Logic Guides, Oxford University Press

Beck JM (1967) Triples, algebras and cohomology. PhD thesis, Columbia University

Busch P, Lahti P, Mittelstaedt P (1996) The Quantum Theory of Measurement, 2nd edn. Springer Verlag 
Dvurečenskij A, Vetterlein T (2001) Pseudoeffect algebras. I. Basic properties. International Journal of Theoretical Physics 40(3):685-701

Foulis D, Bennett M (1994) Effect algebras and unsharp quantum logics. Found Phys 24:1325-1346

Freyd PJ (1964) Abelian categories. Harper \& Row New York

Giuntini R, Greuling H (1989) Toward a formal language for unsharp properties. Found Phys 19:931-945

Harding J (2004) Remarks on concrete orthomodular lattices. International Journal of Theoretical Physics 43(10):2149-2168

Jenča G (2020) Pseudo effect algebras are algebras over bounded posets. Fuzzy Sets and Systems 397:179-185

Jenča G (2015) Effect algebras are the Eilenberg-Moore category for the Kalmbach monad. Order 32(3):439448

Kalmbach G (1977) Orthomodular lattices do not satisfy any special lattice equation. Archiv der Mathematik 28(1):7-8

Kôpka F, Chovanec F (1994) D-posets. Math Slovaca 44:21-34

Ludwig G (1983) Foundations of Quantum Mechanics. Springer-Verlag, Berlin

MacLane S (1971) Categories for the Working Mathematician. No. 5 in Graduate Texts in Mathematics, Springer-Verlag

Mayet R, Navara M (1995) Classes of logics representable as kernels of measures. Contributions to General Algebra 9:241-248

Riehl E (2016) Category theory in context. Courier Dover Publications

van de Wetering J (2021) A categorical construction of the real unit interval. 2106.10094 